\title{
Desktop Computer Virtualization for Improvement Security, Power Consumption and Cost by SBC(Server Based Computer)
}

\author{
Lee Yong Hui ${ }^{1}$ Kim Hwan Seok $^{2}$ and Kim Baek $\mathrm{Ki}^{3}$ \\ ${ }^{1}$ Shinsung University, Chief of Information Service Center, \\ DaehackRo1 Jungmimyun, DanginCity, ChungNam, Korea 343-861, \\ lyhkpi@shinsung.ac.kr \\ ${ }^{2}$ Gangneung-Wonju National University, Dept. Information and \\ Telecommunication Engineering, Korea 220-711, hskim8805@gwnu.ac.kr \\ ${ }^{3}$ Gangneung-Wonju National University, Dept. Information and \\ Telecommunication Engineering, Korea220-711, bkkim@gwnu.ac.kr
}

\begin{abstract}
It is possible for virtualization of desktop to dramatically reduce maintenance costs and improve the security using various virtualization techniques rather than previous desktop environments. Also, with blocking beforehand the information leakage caused by data centralization, it is easy to manage the information security. This desktop virtualization provides creation and duplication of data and standardized desktop environments using easy and fast virtualization works. So, it is possible to improve efficiency, stability, and fusibility of virtualization. In this paper, with the desktop virtualization, the power saving effects are obtained from $65,750(\mathrm{~kW})$ to $7,300(\mathrm{~kW})$, which is from $480(w)$ to $50(w)$ for using one desktop for 8 hours per a day. In addition, the 62 desktops and 62 monitors are combined to one operational server with 62 thin clients. As a result of this, the security is improved greatly by data centralization, which the user can access the main server as a thin client with given space.
\end{abstract}

Keywords: desktop, virtualization, SBC Server host

\section{Introduction}

Recently, companies and governments have been faced with the environment related to electricity usage, maintenance costs, including repair, and internal security leakage for using desktops. The desktop users should access flexibly to the application and data, whenever they need regardless of devices that they use. The existing desktop environment has various limitations related to high maintenance and management costs [1-2, 13], security problems, and constraints of work for using PCs. However, using virtualization of desktops, it is possible to reduce the maintenance costs of previous desktops and to manage the information security because of data centralization. Also, the efficiency, stability, and fusibility will be increased by creation and duplication of easy and fast virtual environment and by standardized desktop environments. The problems of using existing desktops are high energy usage in working environment of using personal desktop computer, leakage and loss of internal information, inefficient managements, and so on. Moreover, it can also be taken place business paralysis during repair time when PC has failure and trouble, permanent loss of working materials when the storage devices malfunction, and danger of leakage of information. The Table 1 shows carbon emission quantity of information technology (IT) area and the proportion of PCs and monitors are estimated $26.8 \%$ and $17.7 \%$, respectively. This tendency has been maintained similar ratio, recently. 
Source: 'Analysis of current energy usage of IT area and research on development of Green IT policy’, NIA.

[unit: Ten thousand tons]

Table 1. Analysis of Current Energy Usage of IT Area

\begin{tabular}{|c|c|c|c|c|c|c|}
\hline Entirety & $\mathrm{PC}$ & Monitor & Printer & Server & IT & Home network \\
\hline \multirow{2}{*}{$\begin{array}{c}1,750 \\
(100 \%)\end{array}$} & $\begin{array}{c}468 \\
(26.8 \%)\end{array}$ & $\begin{array}{c}310 \\
(17.7 \%)\end{array}$ & $\begin{array}{c}453 \\
(25.9 \%)\end{array}$ & $\begin{array}{c}134 \\
(7.7 \%)\end{array}$ & $\begin{array}{c}345 \\
(19.6 \%)\end{array}$ & $\begin{array}{c}40 \\
(2.3 \%)\end{array}$ \\
\hline & \multicolumn{4}{|c|}{$1,365(78.1 \%)$} & \multicolumn{2}{|c|}{$385(21.9 \%)$} \\
\hline
\end{tabular}

\section{Desktop Virtualization}

\subsection{Concepts and Organization}

Desktop virtualization [3-4] is one type of server based computing (SBC) which is technology that user accesses the virtual desktop machines like PC inside data center servers using local devices, usually thin client [5-6], and uses the data, operating system (OS), applications, and so on. Namely, this is a system that can establish dozens of computer in a single central server as a function of computer main body using the virtualization technology and can support work as similar as using the PC. Moreover, it makes virtual desktop environments with combining user environment setting, OS, and applications, depending on user needs and provides these image browsers to users.

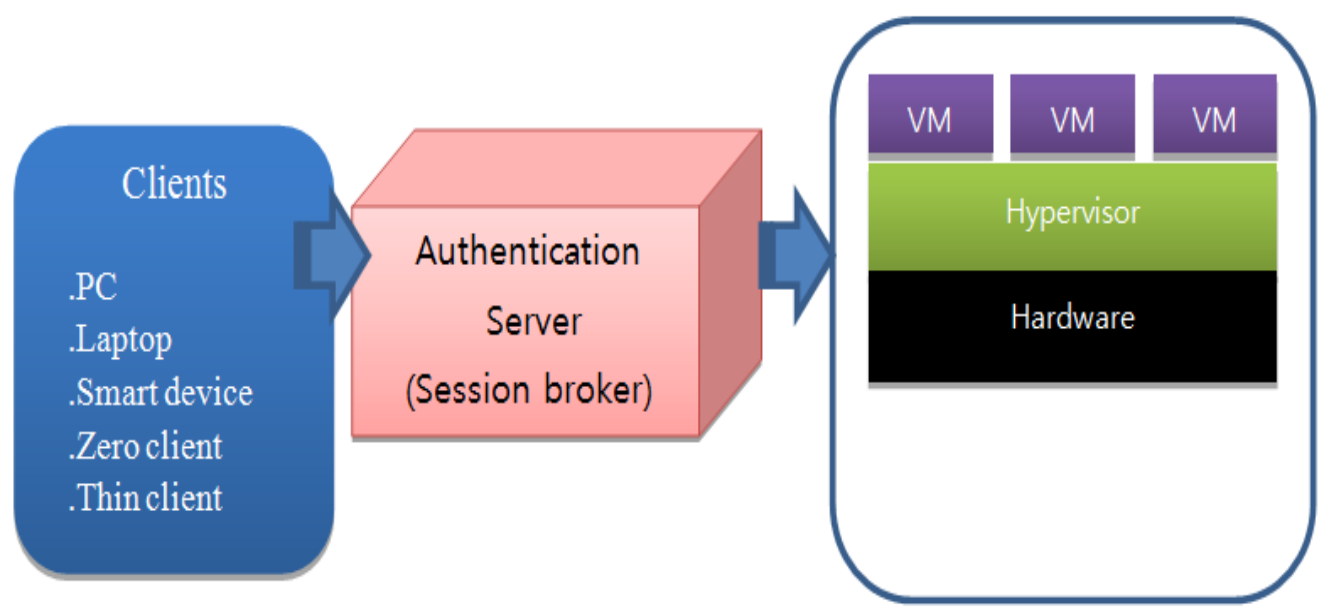

Figure 1. Desktop Virtualization Conceptual Diagram

As shown in the Figure 1, it is needed resources like clients, session broker server, certification server, hypervisor [7, 14], virtual machine [8] operating server, sharing storage, and so on, in the virtual desktop environment.

The following is the needed resources for virtual desktop environment.

- Terminal: Terminal is the device that accesses remotely to the central virtual machine and the software (SW) and hardware (HW) type is existed. SW type can be 
installed and used in the existing PC and HW type can be used as an exclusive device. The HW type terminal divides into zero client and thin client depending on existing own storage. The Table 2 shows the characteristics relative to the type of terminals.

Table 2. Comparison Relative to Types of Terminals

\begin{tabular}{|c|c|c|c|}
\hline Items & Zero client & Thin client & General PC \\
\hline CPU & $\begin{array}{c}\text { No } \\
\text { (Perform only act as } \\
\text { translators })\end{array}$ & Low power CPU & High performance CPU \\
\hline OS & No & Optimum OS & General OS \\
\hline Memory & No & Low(1GB $\downarrow)$ & High(1GB $\uparrow)$ \\
\hline Storage HDD & No & Flash memory & HDD \\
\hline $\begin{array}{c}\text { Power } \\
\text { consumption }\end{array}$ & $5 \mathrm{~W} \downarrow$ & $15 \sim 40 \mathrm{~W}$ & $35 \sim 275 \mathrm{~W}$ \\
\hline Use single & $\begin{array}{c}\text { Impossible } \\
\text { (server required })\end{array}$ & possible & possible \\
\hline
\end{tabular}

- Session Broker Server: Session broker server is the contact point between user terminal and virtualization engine and applies user certification and security policy. This is the device that can store the information which virtual machine transmits to the users with respect to the access request. User terminal get certification through the session broker server and uses the assigned virtual machines. Also, it is conducted a role of security policy between user terminals and virtual machines and a role of maintenance of information for virtual machines.

- Certification Server: Certification server has a responsibility of comprehensive managing the user accounts and of user certification. It is conducted a role of making definitions of small amount relative to the account policy and OS environments and also distribution of it.

- Hypervisor: Hypervisor is the primary factor to execute the virtual machines which has been developing depending on the makers with their own characteristics and the main factor influenced the performance of desktop virtualization.

- Virtual Machines Operating Server: This server has a role to supply the virtualization environment depending on the users with installing OS, hypervisor, and applications.

- Sharing Storage: Sharing Storage is the space to store the image files of virtual machine executing in hypervisor, which contains OS, applications and so on.

\subsection{Classification of Desktop Virtualization}

Desktop virtualization can be divided into client desktop virtualization and the server host virtualization executing in local area and remote area, respectively. The client virtualization also separated into the methods to execute the application directly in the hypervisor without the virtual machines and the methods to drive application in the virtual machines. Server host virtualization is to conduct the application in the remote server and to display through remote display protocol in 
the user terminal screen. The server host virtualization is composed of shared desktop (RDSH) virtualized the session, personal virtual desktop (VDI) as a virtual desktop infrastructure, and blade PC as physical personal desktop. It is executed Window $7 / 8$ or XP desktop in the server of remote data center and expressed the virtual desktop environments through the user terminal for serve host VDI (SHVDI) in server host desktop virtualization. SH-VDI has a non-persistent model to assign the new environment whenever the user log on and a persistent model to give the unique assigned virtual machines to users. Non-persistent model is called stateless desktop which support the similar environments to the users, so it is easy to maintain or manage the image and also it can reduce the storage costs because it can be offer a lot of virtual desktop with simple basic OS images. For persistent model, which is called statefull desktop, it is installed SW freely and kept the consistency of user environment because it supply the unique environments depending on the users. However, the cost is the main problem. In VDI, layered desktop is introduced newly, which has the advantages of both persistent and non-persistent.

\subsection{Structure of Desktop Virtualization}

Infra of desktop virtualization have four elements: clients for mobiles users; broker for connection management; virtual desktop supply server based on hypervisor; and storage supporting user data and virtual images. Client is the user terminal to access their own virtual environment like thin client and PC. The terminals use the basic offered protocol depending on the VDI venders. Broker is storing the information related to the virtual machine structure which is given to users when access is requested. Also, with the user information, they determine: of which server user access to the virtual machine; disperse the load; and conduct the dualization. AD/LDAP connected to broker has a role of user certification which can manage and access the user accounts. Also, it can define the group policy relative to the account policy and window environments and can distribute it en bloc. Hypervisor is the primary factor to execute the virtual machines and there is virtualization engine depending on each vender with their own characteristics; for example, MS Hyper-V, VMWare ESX server, XenServer of Citrix, and OVM of Oracle. The shared storage is the virtual machine OS storage, which store the image files for each OS and can conduct HA and live migration when the failure is taken place in the hypervisor. The relatively high performance storage system is used because of the heavy bottleneck effects when driving a lot of virtual machines.

\subsection{Virtualization Technology}

Currently, Citrix and Vmware occupy $80 \%$ of entire market of desktop virtualization system and Microsoft, Oracle, Quest Software and RedHat, which are open source side, do the market of virtual desktop.

\subsubsection{Citrix XenDesktop}

Citrix took over Xen and have supplied XebDesktop solution, which maintain the top of desktop virtualization market. XendDesktop give the virtual desktop environment based on the XenServer. As XenDesktop provides powerful management tools, it can manage the personal usage area and give the expandable host environment. Also, XenDesktop has functions using hypervisor supporting PC blade and GPU in order to use high quality 3D graphics. The structure of XenDesktop is consist of control module, desktop module, and image module. "Delivery Controller", which is a connection broker, does user certification, manage desktop environment for users, and connect between users and their virtual desktop. Also, it is consisted of the following: XenServer, which is similar to the Hyper-V of 
MS or VMware vSphere; Datastore; provisioning server; web interface; and access gateway in order to approach remote security. XenApp is also included the XenDesktop components, which is used in the remote application streaming. Guest VM or XenDesktop Agent of blade PC is used for direct connection between endpoints and user virtual desktop. The endpoints need Citrix Receiver, which is used for approaching to the virtual desktop or application anywhere and any devices. Protocol of XenDesktop is the independent computing architecture (ICA) and supports more good performance relative to the RFP/RemoteFX in case of having limited bandwidths. XenDesktop 3 applies the high definition experience (HDX) protocol, so it is possible to support VoIP, webcam, 3D, and high quality audio and video with the WAN environment and to improve the user environment. HDX3D Pro supplies the virtual machines as pass through forms in order to service high quality through GPU with sharing physical GPU.

\subsubsection{VMware Horizon View}

After 2000, VMware have lead the server virtualization market and joined VDI market with introducing VDM 2.0 early 2008. Recently, VMware released View5 which can connect vSphere. VDM is a server host virtual machine and changed its name to VMware horizon View. In 2009, VMware introduced PC-over-IP (PCoIP) protocol of Teradici for display protocol. With this, the performance of user remote experience of VMware [9, 15] had been dramatically improved. Also, the local mode is supported in order to use continuous VDI. The structure of VMware is starting View Client and login to View connection server. Combining this server with Windows Active Directory, it is supplied the access authority with respect to VMware vSphere environment, blade or physical PC, or the virtual desktop hosted to the Windows terminal service.

Factors of Horizon View are the following:

- Client Device: physical device that end users use

- View Connection Server: as a broker role of client connection, a role of user certification, and a role of instruction to designated virtual system, physical or blade $\mathrm{PC}$ or windows terminal service server.

- View client: SW for access to the View desktop

- View portal: support to open View desktop inside the browsers

- View Agent: support functions like connection monitoring communicating with View Client, virtual print, View personal setting management, and accessing USB device connected as a local.

- View Administrator: configuration of View Connection Server, management of View desktop, and user certification

- View Composer: It make to establish pool of clone connected upper layer virtual system.

- vCenter Server: support central point to establish, provision, and manage the virtual system of data center with a role of central manager of VMware ESX/ESXi server connected to network.

- View Transmit Server: it is managing and minimizing checked out View desktop in order to user in the end user local system and data transmission between data center.

\subsubsection{Microsoft RDVH-VDI}

MS provide Full desktop session in the Window server starting as a terminal service and virtual desktop as an exclusive program, called RemoteApp, Terminal service of Window server $2008 \mathrm{R} 2$ was changed its name to remote desktop service session host (RDSH) and, once again, to the remote desktop virtualization host 
(RDVH)[10] adding the MS hardware virtual flatform, called Hyper-V. RDVH provides virtual desktop to clients executing window OS. In 2012, MS started to support redesigned complete remote desktop service stack which can make easily manage and install and also to improve the performance of Remote FX which make possible to service high quality in WAN area. MS give remote desktop or application as expanded unique remote desktop protocol (RDP) originated from Remote FX(RFX). In order to support appropriate user experience, the client devices can be driven with Window 7 SP1 or Window 8 with RDP8. MS VDI of Window Server 2012 has the following functions: remote desktop gateway (RDG) which make access to MS VDI; remote desktop web access (RDWA) which permit application access; remote desktop connection broker (RDCB) which make connect between VDI and user when they request; remote desktop session host (RDSH) which is known as terminal services; and remote desktop virtualization host which is MS Hyper-V with agents.

\subsubsection{Oracle VDI}

Oracle provides VDI solution which is belongs to SUN and the following four main components: virtual platform, session management (VDI core), desktop access clients, and storage. VDI core is located in the session management layer can make connect to VMware or MS Hyper-V through application link protocol (ALP). Without this, Dell support virtual desktop or application virtualization through Quest vWorkspace with Provision Networks. Deskton give the cloud desktop virtualization solution like desktop as a service (Daas). LISTEQ also give the VDI solution which can be possible plug and play through BoXedVDI and Virtual Bridges do virtual enterprise desktop environment (VERDE)

\subsubsection{Open Source (Red Hat)}

Red Hat, leading company of open source solution, support virtualization, application, management tools, and service oriented architecture (SOA) solutions based on Red Hat Enterprise Linux, which is high quality OS platform. Red Hat Enterprise Virtualization (RHEV) provides enterprise class open source server and desktop virtualization platforms. There are high performances and many expandability due to supporting Kernel based Virtual Machine (KVM) and various open source components.

\section{Establishment of Desktop Virtualization}

\subsection{H/W Structure}

The background of establishment of desktop virtualization is to eliminate waste of the administrative power due to management of thousands of computer assets, plan of new computer dissemination, maintenance and to improve working environment to protect users with virtualization of PC from harmful environment like heat, noise, and dust from computers. With the reducing power consumptions and getting rid of environmental contamination factors due to abolished PC, the virtualization is needed to make green IT which meets the government policy of "low-carbon green growth". Desktop virtualization HW is needed storages to store desktop resources, and is composed of terminals for usage of each resource. Users are not own the desktop, but monitors and input devices; users use OS and SW accessing the storage and also the personal data space assigned to the storage. Terminal was thin client type without HDD like Figure 2. The number of terminal is 32 for each two spaces, so total 64 terminals were used 


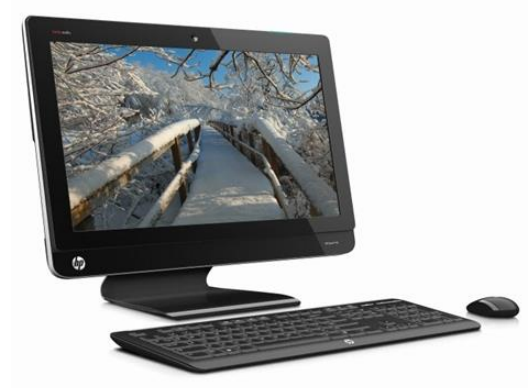

Figure 2. Thin Client Terminal

\section{Figure 3. Personal Computer and Cloud Work Environment Model}

Like Figure 3, the existing PC environment are divided into terminals, operating system, application, and work materials depending on the components, and also the cloud work environment model are done into desktop virtualization, application virtualization, and cloud storage depending on the centralization of components. Desktop Virtualization: The terminal which has a role of monitors and keyboards are left in the office; OS, application, and work materials are all centralized. Application Virtualization: a terminals and OS are left in the office; application and work materials are centralized. Cloud Storage [11, 12]: Only work materials are centralized with the existing PC environment. 

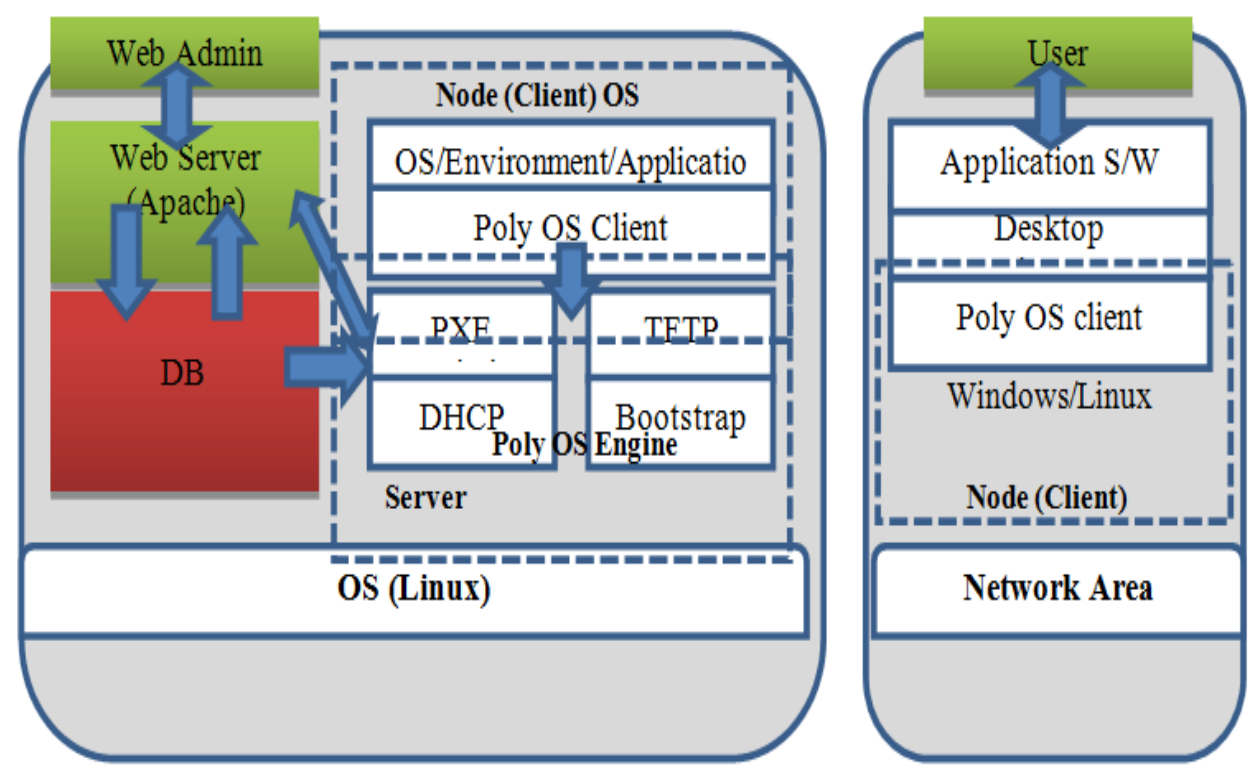

Figure 4. Desktop Virtualized Network Composition

In this paper, all the components of PC (OS, APP) are constituted in the main server, which drive using network and 100\% PC resources (CPU, MEM, Graphic), and also with applying the terminal resources without HDD as shown in Figure 4. These methods make conduct work with downloading the OS and application from main server to terminals at the same time of booting; the generated data is stored in the main server.

\subsection{S/W Composition}

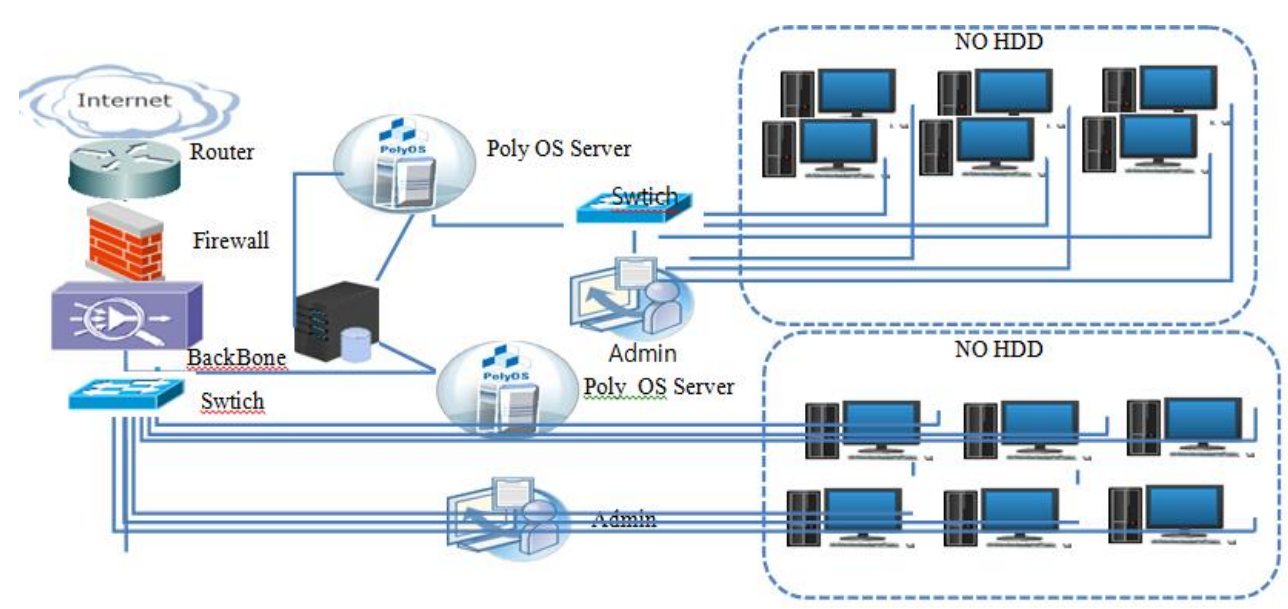

Figure 5. S/W Management and Distribution Diagram 


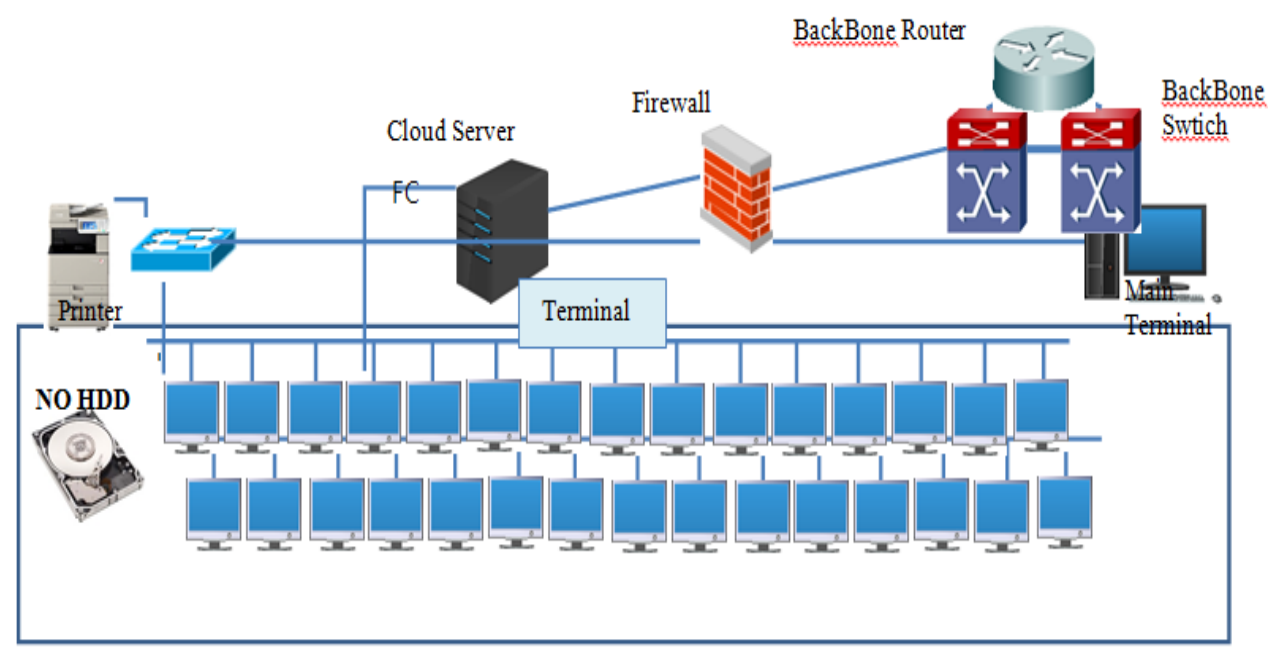

Figure 6. PC Virtualization Diagram

In the user terminals, any $\mathrm{S} / \mathrm{W}$ is not installed and the virtual desktop resources installed in the mounted storage. It is installed in the control server the solution which has a role of generating and deleting virtual desktop. The composition is shown on the Figure 5: to constitute the main control server and storage; to manage each terminal; to store the user data; and to manage and distribute the OS. Figure 6 is PC virtualization diagram of constituted desktop virtualization

\subsection{Results of Constitution}

As a result of constitution of virtualization, it could be earned the Table 3, which is result before and after virtualization. Through desktop virtualization, the power saving effects was obtained from $65,750(\mathrm{~kW})$ to $7,400(\mathrm{~kW})$ per year if using 8 hours per day, which reduced from $480(w)$ to $50(w)$ for one desktop. In terms of costs, $90 \%$ is saving from $\$ 4,650$ to $\$ 480$. Through virtualization, the convenience of management has been secured due to centralized managements, and also the work continuity has been guaranteed because of protecting data loss with separated important data storage. It has been achieved that the security is improved by protection of leakage of data to external. In terms of costs, the cost saving effects was obtained relative to the power saving, number of usage year, maintenance, and replacement duration.

Table 3. Power Consumption before and after Virtualization 


\begin{tabular}{|l|r|r|r|r|}
\hline \multirow{2}{*}{ Items } & \multicolumn{2}{|c|}{ Before virtualization } & \multicolumn{1}{c|}{$\begin{array}{c}\text { After } \\
\text { virtualization }\end{array}$} & Blank \\
\cline { 2 - 4 } & \multicolumn{1}{|c|}{ Desktop } & Monitor & Thin client & \\
\hline Qty(ea) & 50 & 50 & 50 \\
\hline Power(w) & 450 & 30 & 50 \\
\hline $\begin{array}{l}\text { Consumption power } \\
\text { / 1day(w) }\end{array}$ & 180,000 & 12,000 & 20,000 \\
\hline $\begin{array}{l}\text { Consumption power } \\
\text { / 1year(w) }\end{array}$ & $65,700,000$ & $4,380,000$ & $7,300,000$ \\
\hline Change unit to (Kw) & 65,700 & 4,380 & 7,300 \\
\hline Total cost & & $\$ 4,650$ & $\$ 480$ \\
\hline
\end{tabular}

\section{Conclusion}

The existing PC based work environment has problems of energy waste, information leakage and failure, and inefficient management. Carbon emission quantity depending on usage of PC is estimated $27 \%$ of total IT area. In this paper, the leakage prevention effect for internal administration information is obtained by en bloc application of server based centralized security policy through desktop virtualization. In terms of power consumption, $90 \%$ of cost saving was earned relative to the normal computer. Also, the user work environment is improved and the efficiency is dramatically progressed with eliminating noise, dust, electromagnetic wave. Through this research, although the market has been growing with the advantage of virtual desktop; for example, power saving, high security, and low TCO, it has been deduced that the compatibility between products are the major problem due to supporting independent solution using the unique platform and protocol depending on each vender. The international standardized activity has been progressed in ITU-T relative to desktop virtualization, so DaaS standard is prospected to complete. Henceforth, because various type of clouding service is established based on virtual desktop, it is needed to be researched continuously on developing the core technology of desktop virtualization with open source S/W and also the standardization work.

\section{References}

[1] L. Y. Hui and K. H. Seok, "A Study of Savings of Power Consumption and Server Space through Integrated Virtualization of UNIX Servers", IJMUE, SCOPUS, ISSN 1975-0080, vol. 9, no. 9, (2014) September, pp. 255-260.

[2] R. Brown, "Report to Congress on Server and Data Center Energy efficiency", Public Law 109-431, Lawrence Berkely National Lab, (2008).

[3] D. Oliveira, V. Chaves, MOTA, A. De Assis and L. T. Moreira, "Impacts of Application Usage and Local Hardware on the Throughput of Computer Networks with Desktop Virtualization", American Journal of Applied Sciences, vol. 10, Issue 1, (2013), pp. 117-122.

[4] J. S. Min, C. W. Hyuk and K. W. Young, "Client Rendering Method for Desktop Virtualization Services”, ETRI Journal, vol. 35, Issue 2, (2013), pp. 348-351.

[5] L. Deboosere and B. Vankerirsbilck, "Cloud-Based Desktop Services for Thin Clients", Internet Computing, IEEE, ISSN 1089-7801, vol. 16, Issue 6, (2011), pp. 60-67.

[6] D. Mega, M. Hiebel and C. Knermann, "Comparison of Two ICT Solutions: Desktop PC versus Thin Client Computing", IJLCA, vol. 18, Issue 4, (2013) May, pp. 861-871.

[7] V. Amit, "Design Implementation and Verification of an Extensible and Modular Hypervisor Framework", Security and Privacy (SP), IEEE Symposium on IEEE, (2013), pp. 430-444.

[8] W. Jing, Z. F. Zhi, D. Y. Dan, "Design on Virtual Machine Frame of Cloud Computing Platform", Advanced Materials Research, vol. 834-836, (2014), pp. 1832-1835.

[9] E. Bugnion, "Bringing Virtualization to the x86 Architecture with the Original vmware Workstation", 
ACM Transactions on Computer Systems (TOCS), vol. 30, Issue 4, (2012), pp. 12.

[10] X. Yunpeng, "A Metadata-driven Cloud Computing Application Virtualization Model”, Journal of Computers, vol. 8, no. 6, (2013), pp. 1571-1579.

[11] Y. Wenbin and L. Liang, "A Selection Algorithm of Service Providers for Optimized Data Placement in Multi-Cloud Storage Environment", Intelligent Computation in Big Data Era. Springer Berlin Heidelberg, vol. 503, (2015), pp. 81-92.

[12] L. Hong-Chang, A. Hyung-Bae and L. Myung-Joon, "Operation Atomicity and Storage Replication in a Collaborative Middleware Based on Cloud Storage", Future Information Technology. Springer Berlin Heidelberg, vol. 309, (2014), pp. 833-840.

[13] L. Y. Hui and K. H. Seok, "Virtualization of Desktop for Improvement of Maintenance Cost Saving", Advanced Science and Technology Letters (Information Technology and Computer Science, vol. 85, (2015), pp. 33-37.

[14] D. Perez-botero, J. Szefer, R. B. Lee, "Characterizing Hypervisor Vulnerabilities in Cloud Computing Servers", International Workshop on Security in Cloud computing. ACM, (2013), pp. 3-10.

[15] P. Padala, "Resource Management in VMware Powered Cloud: Concepts and Techniques", Parallel \& Distributed Processing (IPDPS), IEEE 27th International Symposium on IEEE, (2013), pp. 581-581.

\begin{abstract}
Authors
Lee Yong Hui, He received the BS, MS and $\mathrm{PhD}$ in Electronic Engineering from CheongJu University, CheongJu, Rep. of Korea, in 1989, 1991 and 2001, respectively. He worked at HYNIX Semiconductor ULSI LAB from 1995 to 2002. Since 2002, he has been dept, of Computer Application and Chief of Information Service Center at Shinsung University, Dangin, Rep. of Korea. His current research interests include computer control, microprocessor, web programming, RFID, server, network and information security.

Kim Hwan Seok, received the BS, MS and $\mathrm{PhD}$ in Electronic Engineering from CheongJu University, CheongJu, Rep. of Korea, in 1988, 1990 and 2008, respectively. Since 1992, he has been dept, of Information and Telecommunication Engineering, GangneungWonju National University, WonJU, Rep. of Korea. His current research interests include VLSI \& CAD, smart product, smart healthcare and application programming. Also, he is corresponding author in this paper.

Kim Baek Ki, He received the B.S. degree in electronic Engineering from the Kyunghee University in 1985, and the M.S. degree in electronic Engineering from the Kyunghee University in 1987. He received the Ph.D. Degree in electronic Engineering from the Kyunghee University in 1997. His research interests are the signal processing and multimedia and smart health care and applications of Korea. His current research interests include computer control, microprocessor, web programming, RFID, server, network and information security.
\end{abstract}


International Journal of Security and Its Applications Vol. 9, No. 5 (2015) 